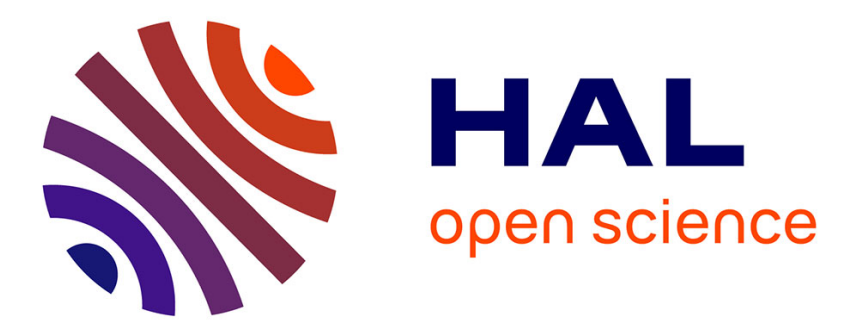

\title{
Hot and Cold Charge-Transfer Mechanisms in Organic Photovoltaics: Insights into the Excited States of Donor/Acceptor Interfaces
}

\author{
Daniele Fazzi, Mario Barbatti, Walter Thiel
}

\section{- To cite this version:}

Daniele Fazzi, Mario Barbatti, Walter Thiel. Hot and Cold Charge-Transfer Mechanisms in Organic Photovoltaics: Insights into the Excited States of Donor/Acceptor Interfaces. Journal of Physical Chemistry Letters, 2017, 8 (19), pp.4727-4734. 10.1021/acs.jpclett.7b02144 . hal-02288766

\section{HAL Id: hal-02288766 \\ https://hal-amu.archives-ouvertes.fr/hal-02288766}

Submitted on 16 Sep 2019

HAL is a multi-disciplinary open access archive for the deposit and dissemination of scientific research documents, whether they are published or not. The documents may come from teaching and research institutions in France or abroad, or from public or private research centers.
L'archive ouverte pluridisciplinaire HAL, est destinée au dépôt et à la diffusion de documents scientifiques de niveau recherche, publiés ou non, émanant des établissements d'enseignement et de recherche français ou étrangers, des laboratoires publics ou privés. 
Hot and cold charge transfer mechanisms in organic photovoltaics: insights into the excited states of donor/acceptor interfaces.

Daniele Fazzia, Mario Barbattib and Walter Thiel ${ }^{a}$

a) Max-Planck-Institut für Kohlenforschung, Kaiser-Wilhelm-Platz 1, D-45470 Mülheim an der Ruhr, Germany.

b) Aix Marseille Univ, CNRS, ICR, Marseille, France.

\section{ABSTRACT}

The evolution of the excited-state manifold in organic D/A aggregates (e.g. the prototypical P3HT/PCBM) is investigated through a bottom-up approach via first-principles calculations. We show how the excited-state energies, the charge transfer (CT) states, and the electron-hole density distributions are strongly influenced by the size, the orientation and the position (i.e. on-top vs. on-edge phases) of P3HT/PCBM domains. We discuss how the structural order influences the excited-state electronic structure, providing an atomistic interpretation of the photophysics of organic blends. We show how the simultaneous presence of on-top and on-edge phases does not alter the optical absorption spectrum of the blend but does affect the photophysics. Photovoltaic processes such as $i$ ) the simultaneous charge generation obtained from hot and cold excitations, ii) the instantaneous and delayed charge separation, and iii) the pump-push-probe charge generation, can be reconsidered based on our study. 


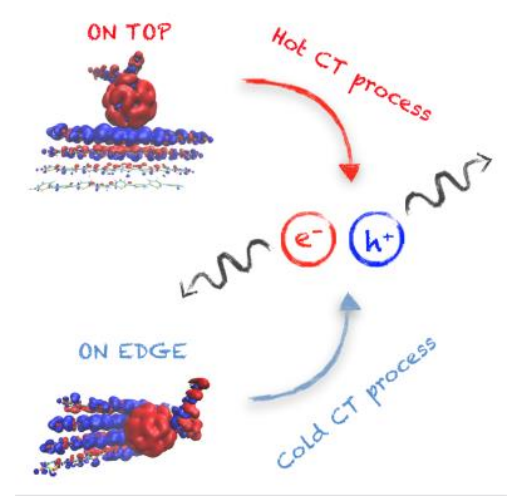

Light-to-current conversion processes lie at the base of any photovoltaic technology. ${ }^{1}$ Regardless of the active materials, a heuristic approach would suggest that the easier it is to transfer the excitation energy into free charges, the higher would be the power conversion efficiency of the solar cell. However, exciton dissociation has to compete with thermalization mechanisms that decrease the odds of charge generation. ${ }^{2}$ This problem is particularly severe in organic photovoltaics (OPV), ${ }^{3}$ affecting the global efficiency of solar cells. ${ }^{4} \mathrm{~A}$ key point to improve OPV performance is to understand the photo-induced mechanisms in the region where excitons split into charges, i.e. at the donor/acceptor (D/A) interfaces. ${ }^{5}$ Assessing the nature of the excited states at D/A interfaces is still one of the major challenges, and thus crucial, in organic electronics and photovoltaics. ${ }^{6}$ The aim of this work is, therefore, to investigate, via first-principles calculations, the nature of the excited states in D/A clusters. We show how the excited-state energies, the charge transfer (CT) states, and the electron-hole density distributions are influenced by the size, the orientation, and the position of polymer/fullerene-like domains. We discuss evidence of how structural order influences the excited-state electronic structure in D/A 
interfaces, provide an interpretation of the OPV photophysics, and suggest potential ways to boost productive charge transfer processes.

The photophysics ruling charge generation in OPV is still a much-debated topic, leading to conflicting descriptions. Some accepted interpretations have emerged in recent years; amongst those the most relevant are briefly listed here: 1) Free charges can be generated independently from the photon excitation energy (either below or above the optical gap). ${ }^{7}$ 2) High-energy excitations lead to electron transfer ${ }^{8}$ via the population of hot CT states; ${ }^{9}$ however, these states are not necessarily responsible for the generation of free charges. ${ }^{10} 3$ ) Delocalized electronic states facilitate charge separation, by lowering the electron-hole bound-potential barrier. ${ }^{8} 4$ ) Long-range electron-hole dissociation may occur through tunnelling toward delocalized states, ${ }^{11}$ before the excitons actually reach the interface, ${ }^{12}$ or 5 ) through direct optical population of D/A states featuring CT character. ${ }^{13}$ Furthermore, specifically for molecular based donor-acceptor-donor systems, ${ }^{14}$ geometry relaxation can lead to competition between energy and charge transfer events. ${ }^{15}$

Recent ultrafast transient absorption spectroscopy experiments were used to determine the time scales and the spatial distribution of photogenerated charge pairs in OPV cells. ${ }^{16}$ Notably, long-range charge separation was found to occur within the time scale of thermalization, invoking early branching between free and bound charges across the organic interface. ${ }^{16 \mathrm{~b}}$ These observations suggest a complex energetic scenario, which should consider both the excited-state character $^{17}$ and the electrostatic potential across the interface. ${ }^{18}$

Remarkable insights into the nature of the excited states at D/A interfaces (e.g., P3HT/PCBM, ${ }^{19}$ PCPDTBT/PCBM, ${ }^{9}$ pentacene $/ \mathrm{C} 60^{20}$ or $\mathrm{DCV} n \mathrm{~T} / \mathrm{C} 60^{21}$ ) were 
achieved by several research groups. ${ }^{19 a}$, 20a, 20b, 21-22 Density functional theory (e.g., DFT/TDDFT), 19, 20a, 23 many-body Green's functions theory (e.g., GW approximation), ${ }^{22 a}, 24$ and ab initio methods (e.g., $\left.\mathrm{ADC}(2)\right)^{25}$ were applied on minimal model systems to calculate the low-lying excited states (usually the first few) in D/A molecular complexes. ${ }^{26}$ Attention was paid mainly to the energy of the first CT state with respect to the active (i.e. dipole-allowed) excited state. It is generally assumed that charge transfer can occur if $\mathrm{E}(C T)<\mathrm{E}$ (active), in an exothermic process $(\Delta \mathrm{E}<0)$. However, the focus on minimal structural models and a small number of computed excited states might not fully catch the complex photophysics of OPV blends.

Here, we systematically investigate the evolution of the excited-state manifold in D/A aggregates (for the prototypical P3HT/PCBM system) through a bottomup approach, by increasing the size of $\mathrm{D}$ and A domains and by changing their relative orientations and configurations. Homogeneous (A/A, D/D) and heterogeneous (D/A) clusters are considered, as shown in Figure 1.
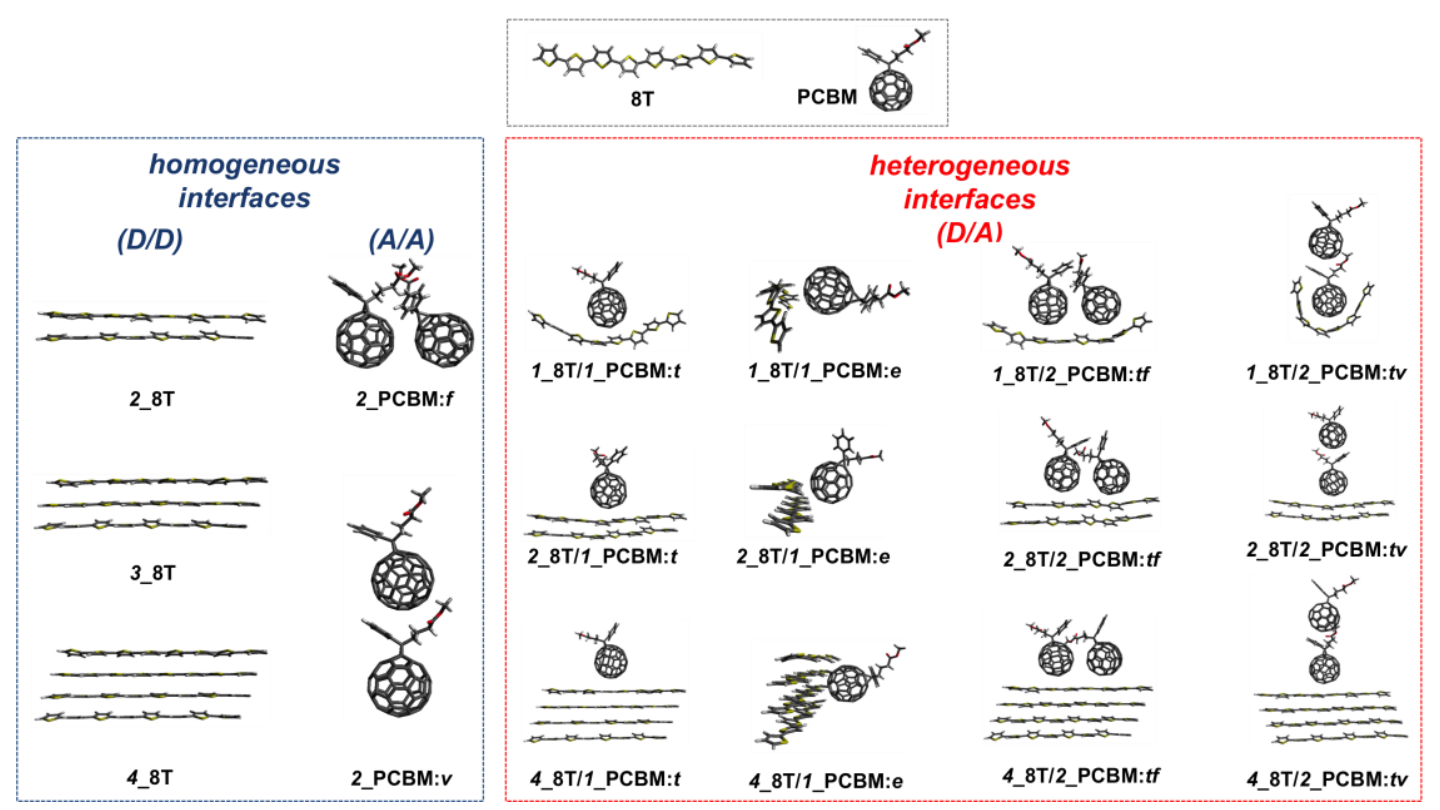
Figure 1 Molecular structures investigated in this work. Top panel: isolated oligothiophene chain (octamer, 8T) and PCBM. Left panel: homogeneous domains of donors (D/D) or acceptors (A/A). Donors domains include 2, 3 or 4 8T chains, respectively, while acceptor domains consist of 2 PCBM in face-to-face $(f)$ or vertical $(v)$ configuration. Right panel: heterogeneous interfaces (D/A) are classified as $n_{-} 8 \mathrm{~T} / m_{-}$PCBM: $i$, with $n$ number of $\pi-\pi$ 8T, $m$ the number of PCBM and $i$ an index describing the configuration of PCBM with respect to the donor cluster, i.e. on-top $(t)$, on-edge $(e)$, on-top face-to-face $(t f)$ or on-top vertical $(t v)$. Each structure was optimized at the $\omega$ B97X-D/6-31G* level (gas phase).

Donor domains are classified as $n_{-} 8 \mathrm{~T}$, with $n$ the number of $\pi-\pi$ stacked chains, each consisting of 8 monomers. Acceptor domains are classified as 2_PCBM: $f$ or 2_PCBM: $v$, representing two PCBM either in a face-to-face $(f)$ or vertical ( $v$ ) arrangement (see Figure1). D/A interfaces are classified as $n \_8 \mathrm{~T} / m_{-}$PCBM:i, with $n$ the number of $\pi-\pi$ stacked thiophene-octamers, $m$ the number of PCBM and $i$ the relative position of PCBM with respect to the polythiophene cluster $(i=t, e, t f$, $t v$ with $t$ for on-top, $e$ for on-edge, $t f$ for on-top face-to-face, $t v$ for on-top vertical, see Figure1). Each structure was optimized (i.e. stable stationary point) in the ground state (gas phase) at the DFT level using a range-separated functional with dispersion corrections included, i.e. $\omega B 97 X-D / 6-31 G^{*}$. All DFT calculations were performed using the Gaussian09 package ${ }^{27}$.

Vertical excitation energies were computed at the TD-DFT level, both in the gas phase and with the conductor polarizable continuum model (C-PCM, with a dielectric constant $\varepsilon=3.0$ as commonly accepted for organic semiconductors ${ }^{20 a}$ ). For 1_8T/1_PCBM:t, the geometry of the first excited singlet state $\mathrm{S}_{1}$ was optimized at the TD-DFT level in order to assess the equilibrium structure and state character (see Supporting Information). Detailed investigations by Brédas et al., ${ }^{20 a}, 23$ Kronik et al., ${ }^{28}$ and Kümmel et al. ${ }^{29}$ have established that suitably 
adapted range-separated functionals ${ }^{30}$ describe the energy and character of the excited states reasonably well, both in the condensed phase and at donor/acceptor interfaces. ${ }^{17}$ Accordingly, excitation energies were also computed by tuning the range-separation parameter $\omega$ (see Supporting Information). A shift of $\sim 0.10 \mathrm{eV}$ in the vertical energies was found when using the optimized $\omega$ value $\left(0.17 \text { bohr }^{-1}\right)^{20 a}$ instead of the default value $\left(0.20 \mathrm{bohr}^{-1}\right)$ (see Supporting Information); however, the state order remained the same. Moreover, regardless of the chosen $\omega$ value or the presence of a polarizable continuum, the physical picture, the photovoltaic mechanisms, and the general considerations drawn here (vide infra) remained unchanged.

Excited states were classified as localized on the donor $(L D)$ or on the acceptor ( $L A)$ domain, as delocalized over the interface (deloc) or as involving charge transfer $(C T)$ from one domain to the other. Amongst different ways to classify excitons in molecular based systems, ${ }^{31}$ our state classification procedure is based on the analysis of the electron density difference $(\Delta \rho)$ between the investigated excited state and the ground state $\left(\Delta \rho=\Delta \rho^{\text {excited state }}-\Delta \rho^{\text {ground state }}\right)$, as described in Refs. 17 and 32.

Detailed results for the vertical excitation energies of homogeneous interfaces (A/A and D/D) and their evolution with increasing cluster size are reported in Figure SI1 of the Supporting Information. Briefly, for donor clusters, we observe a splitting of the first dipole-allowed excited state when going from one oligomer (1_8T) to four $\pi$-stacked oligothiophene chains (4_8T), which gives rise to the formation of a mini-band. The computed electronic spectra of the donor cluster 4_8T exhibit two main absorption bands: a low-energy band (LE $=2.5-3.2 \mathrm{eV}$ ) and a high-energy band ( $\mathrm{HE}=3.5-4.0 \mathrm{eV}$ ). The corresponding excited states are 
delocalized, spreading either over two neighboring chains or over the entire cluster (vide infra). For acceptor domains ( $n_{-}$PCBM), we find no significant changes in the excited states when going from one to two PCBMs (Figure SI1). Figure 2 shows the evolution of the energy and character of the excited states and the calculated absorption spectra for relevant (vide infra) D/A model clusters, amongst those reported in Figure 1. Depending on the donor domain size and the relative positions of the acceptor, the energy and character of the excited states change, thus indicating specific structure-property relationships between the local interfacial geometry and the photophysics of the organic interfaces. At the level of the molecular dimer (1_8T/1_PCBM), a model commonly adopted when describing OPV interfaces quantum-chemically, ${ }^{2 c}, 26$ the position of PCBM affects the relative energy of the dipole-allowed excited state and the first CT state ( $\underline{\text { Figure } 2}$ and $\underline{\text { Table } 1)}$. 


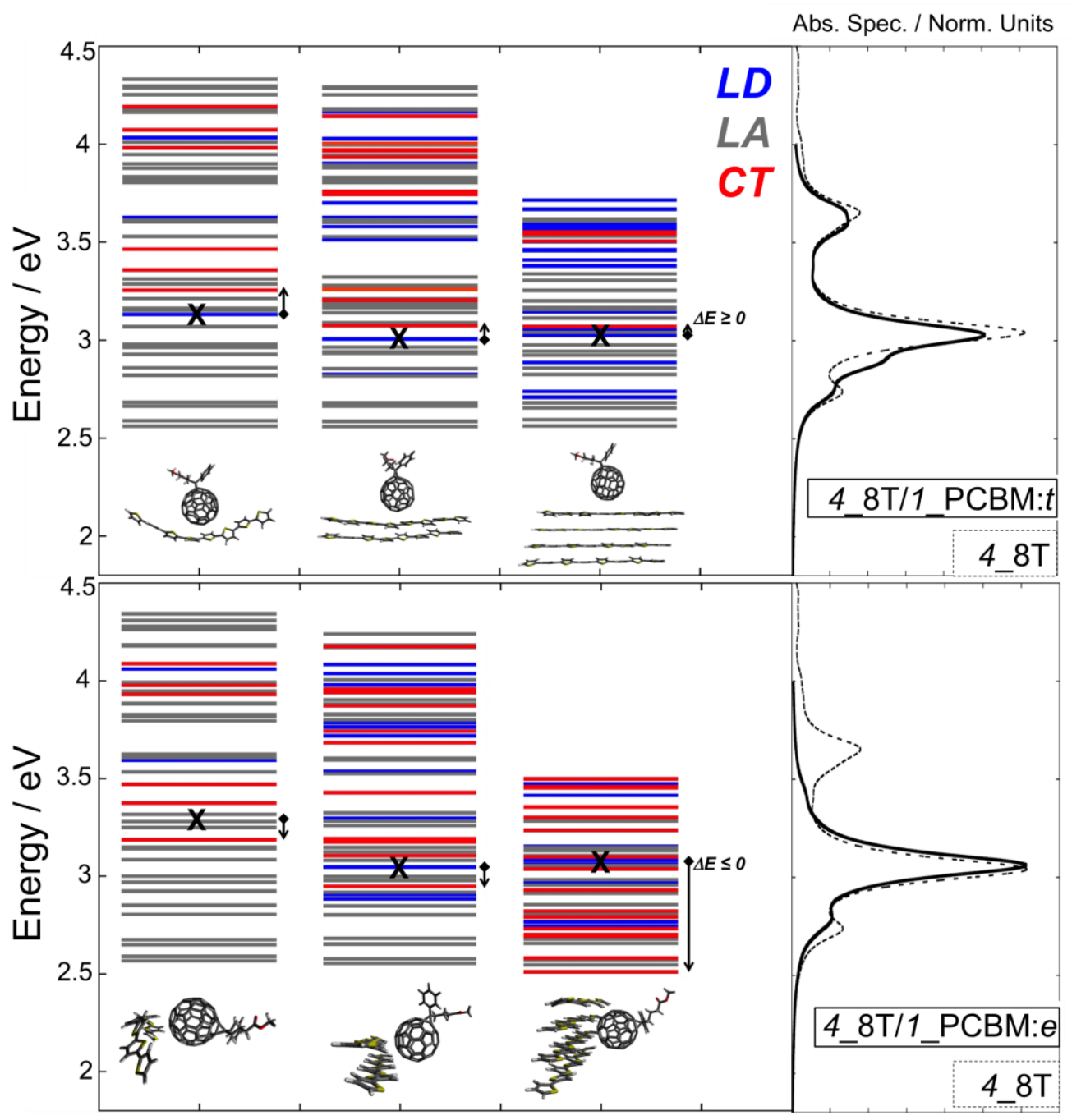

Figure 2 Vertical excitation energies computed at the TD- $\omega B 97 X-D / 6-31 G^{*}$ level for different D/A clusters, namely 1_8T/1_PCBM, 2_8T/1_PCBM and 4_8T/1_PCBM, with PCBM in on-top (top panel) or on-edge (bottom panel) configuration. Right-hand side: comparison between the absorption spectra of big D/A clusters (4_8T/1_PCBM) and a pure donor domain (4_8T). Absorption spectra were calculated as a convolution of Lorentzian functions on top of the computed TD-DFT energies and oscillator strengths. Excited states are classified as: localized on the donor ( $L D$, blue), localized on the acceptor ( $L A$, grey), or charge-transfer from the donor to the acceptor $(C T$, red). $\mathbf{X}$ identifies the lowest dominant bright state (i.e., active) and the arrows identify its energy separation ( $\triangle \mathrm{E}=\mathrm{E}(C T)$ - $\mathrm{E}$ (active) ) from the closest CT state; $\Delta \mathrm{E} \geq 0$ for on-top configurations and $\Delta \mathrm{E} \leq 0$ for on-edge configurations.

Table 1 TD-DFT vertical excitation energies and oscillator strengths for different cases, namely: isolated 8T and PCBM, D/A molecular dimer (1_8T/1_PCBM), 2_8T/1_PCBM, 4_8T/1_PCBM and 4_8T/2_PCBM in different configurations (as specified in the notation). The brightest LD state in the first absorption band and the first CT state are reported in each case (for comparison, see also Figure 2). 


\begin{tabular}{l|llll}
\hline Structure & $\mathrm{E}(\mathrm{LD}) / \mathrm{eV}$ & $f$ & $\mathrm{E}(\mathrm{CT}) / \mathrm{eV}$ & $f$ \\
\hline 8T & 3.17 & 2.88 & & \\
PCBM & 2.55 & 0.00 & & 0.02 \\
\hline 1_8T/1_PCBM:t & 3.13 & 1.05 & 3.25 & 0.00 \\
1_8T/1_PCBM:e & 3.27 & 0.96 & 3.18 & 0.06 \\
\hline 2_8T/1_PCBM:t & 3.00 & 4.67 & 3.07 & 0.00 \\
2_8T/1_PCBM:e & 3.04 & 3.91 & 2.94 & 0.34 \\
\hline 4_8T & 3.03 & 5.25 & & 0.01 \\
\hline 4_8T/1_PCBM:t & 3.02 & 4.25 & 3.07 & 0.00 \\
4_8T/1_PCBM:e & 3.06 & 5.72 & 2.51 & 0.24 \\
\hline 4_8T/2_PCBM:tf & 3.02 & 3.94 & 2.97 & \\
4_8T/2_PCBM:tv & 3.03 & 5.19 & 3.05 & \\
\hline
\end{tabular}

In 1_8T/1_PCBM:t, the first optically active state (localized on the donor, $L D$ ) is at $3.13 \mathrm{eV}$, being $0.12 \mathrm{eV}$ lower than the first CT state $(3.25 \mathrm{eV})$, see Table 1. In 1_8T/1_PCBM:e, the first bright state (delocalized over the interface, deloc) is at $3.27 \mathrm{eV}$, lying $0.08 \mathrm{eV}$ higher than the first CT state $(3.18 \mathrm{eV}$ ) (in Figure 2 the brightest low-energy states are marked with $\mathbf{X}$ ). In these minimal models, the charge transfer process from the polymer to the PCBM thus seems to be thermodynamically allowed in the Franck-Condon (FC) region for the on-edge configuration $(\Delta \mathrm{E}=-0.08 \mathrm{eV})$, but not for the on-top counterpart $(\Delta \mathrm{E}=+0.12 \mathrm{eV})$. Increasing the size of the donor domain, from 2_8T/1_PCBM to 4_8T/1_PCBM, does not affect the computed state ordering in both configurations (Figure 2). However, the energy and the classification of the excited states substantially differ from the dimer model. For clusters with PCBM in the on-top configuration, the energy of the first CT state decreases by increasing the donor domain, shifting from $3.25 \mathrm{eV}$ in 1_8T/1_PCBM:t to $3.07 \mathrm{eV}$ in 4_8T/1_PCBM:t (see $\underline{\text { Table }}$ 1). Although the population of the first CT state remains thermodynamically unfavorable in the $\mathrm{FC}$ region, $\mathrm{E}(C T)>\mathrm{E}$ (active) (Table 1) , with $\Delta \mathrm{E}=+0.05 \mathrm{eV}$, the CT state lies within the optical absorption band (quasi in resonance with the dipole-allowed state at $\mathrm{E}=3.02 \mathrm{eV}, f=4.25$, Figure 2). In this energetic scenario, 
a direct optical population of CT states via laser pumping would seem possible in the FC region, eventually evolving toward productive charge-separated states. We note that the excited states at the bottom of the band are localized on the PCBM (Figure 2) and have very low oscillator strength $(f<0.05)$. Population of these states, for instance via internal conversion (IC) from high-energy states, ${ }^{10}$, 33 might lead to localization processes that impede charge separation. ${ }^{34}$

For clusters with PCBM in the on-edge configuration (n_T/1_PCBM:e), the evolution of the excited-state energies and characteristics differs from the on-top cases. When increasing the donor domain size $(n=2,4)$, CT states increase in number, and their energies decrease until they reach the bottom of the excited state band. For the largest cluster, 4_8T/1_PCBM:e, the lowest CT state is at 2.51 $\mathrm{eV}$, a decrease of $0.67 \mathrm{eV}$ with respect to the dimer model (see Figure 2 and Table 1). The strongest dipole-allowed state, localized on the D domain, is at $3.06 \mathrm{eV}(f=5.7)$. The enthalpic driving force for populating the lowest CT state is $\Delta \mathrm{E}=-0.55 \mathrm{eV}$, implying a very favorable thermodynamic process in the $\mathrm{FC}$ region.

We also considered larger D/A clusters, pushing the limits of our computational capabilities at the TDDFT level. Examples of optimized groundstate structures are reported in Figure 1 and in the Supporting Information, namely $n_{-} 8 \mathrm{~T} / 2 \_$PCBM:i, with two PCBM on-top face-to-face ( $t$ ) or on-top vertical $(t v)$ positions. The presence of a second PCBM neither alters the excited-state picture, as previously drawn for smaller clusters, nor significantly changes the energy or the character of the excited states (see $\underline{\text { Table } 1}$ and Supporting Information). Similar to the case with one PCBM in on-top configuration, D/A interfaces with multiple PCBMs have CT states in the FC region that lie within the 
optical gap, in quasi-resonance with the dipole-active state (see classifications in the Supporting Information).

In Figure 3 we compare the excited-state energies, the electron-hole density maps, and the calculated absorption spectra for three relevant cases of polymer/fullerene blends: the pure polymer donor domain, modelled as 4_8T cluster, and two kinds of D/A interfaces modeled as oligothiophene clusters with one PCBM in the on-top (4_8T/1_PCBM:t) or the on-edge (4_8T/1_PCBM:e) configuration. In the donor cluster (4_8T), the LE absorption band arises mainly from the strongest dipole-allowed transition in the FC region to the $\mathrm{S}_{4}$ state $(3.03$ $\mathrm{eV}, \mathrm{f}=5.25$, Table 1), in which the exciton is spatially delocalized over the cluster, as can be seen from the analysis of the electron-hole density map ( $\underline{\text { Figure 3) }}$. Other low-energy states of 4_8T reported in Figure 3 are $S_{5}(3.06 \mathrm{eV}$, $f=1.05), S_{3}(2.95 \mathrm{eV}, f=0.00), S_{2}(2.73 \mathrm{eV}, \mathrm{f}=1.11)$, and $S_{1}(2.71 \mathrm{eV}, \mathrm{f}=0.00)$. In each of these states, the electron-hole density distribution is delocalized over the cluster. 


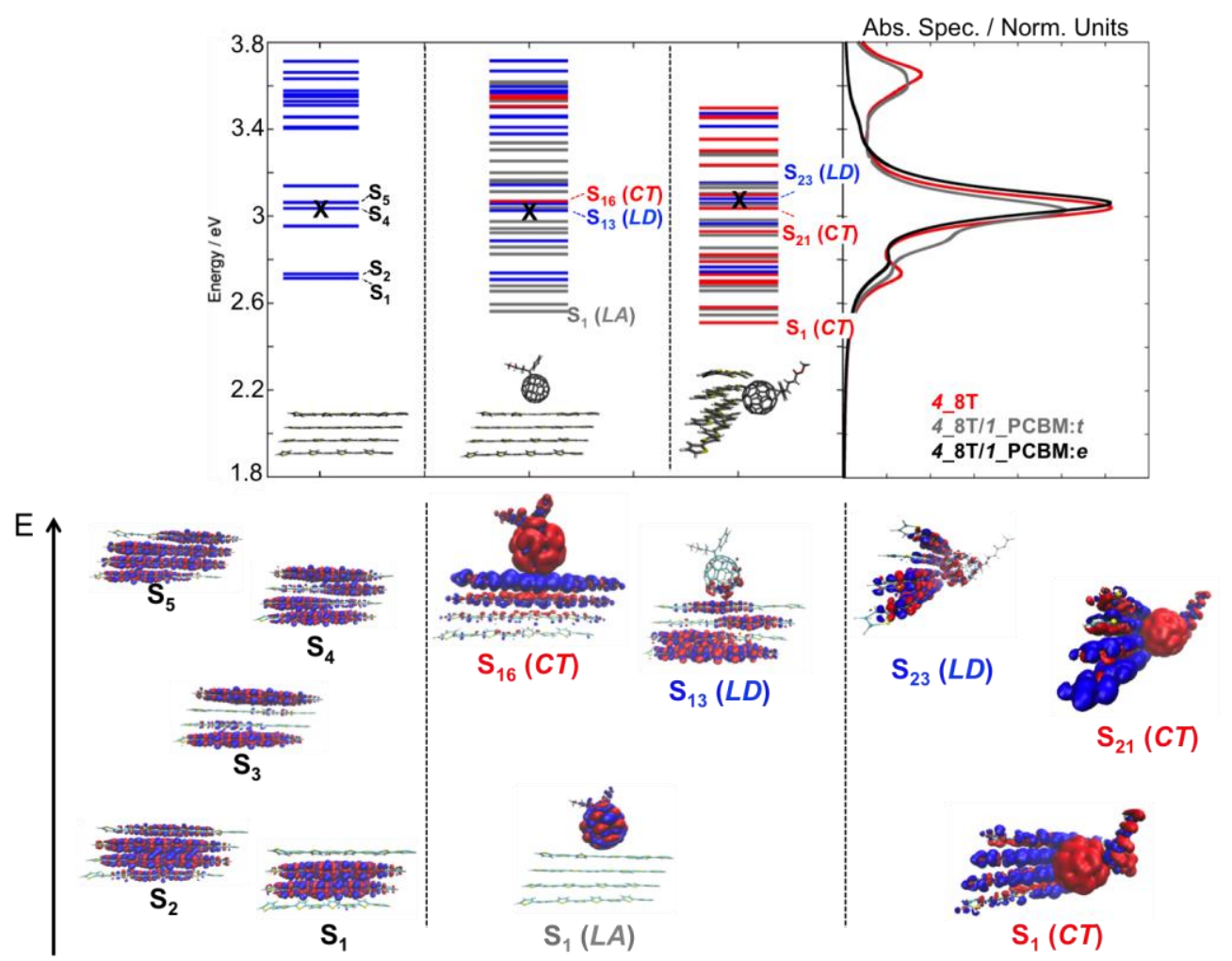

Figure 3 Comparison between three relevant cases: donor cluster - 4_ 8T, D/A cluster in the on-top configuration - 4_8T/1_PCBM:t, and D/A cluster in the onedge configuration - 4_8T/1_PCBM:e. Top panel: vertical TD-DFT energies, state classifications (blue $-L D$, grey $-L A$, red $-C T$ ), absorption spectra (red for 4_8T, grey for 4_8T/1_PCBM:t, black for 4_8T/1_PCBM:e) and optimized structures for each case. Bottom panel: electron-hole density maps for $L D$ and $C T$ states that are relevant for understanding the photovoltaic processes (electron - red, hole blue).

In the D/A cluster with PCBM in the on-top configuration (4_8T/1_PCBM:t) the strongest transition is to $\mathrm{S}_{13}(3.02 \mathrm{eV}, \mathrm{f}=4.25)$, in which the electron-hole density distribution is mainly located on the donor chains, therefore classified as $L D . \mathrm{S}_{13}$ corresponds to the bright state $\mathrm{S}_{4}$ of the donor domain 4_8T. The first CT state $\left(\mathrm{S}_{16}\right)$ in 4_8T/1_PCBM:t lies slightly above $\mathrm{S}_{13}\left(\mathrm{~S}_{16}=3.07 \mathrm{eV}, \mathrm{f}=0.3\right.$, Table 1) and is characterized by a net electron-hole separation, with the hole being delocalized over the oligothiophene chains closest to the PCBM, while the electron is on the PCBM. The first excited state at the bottom of the band $\left(\mathrm{S}_{1}=\right.$ $2.56 \mathrm{eV}, \mathrm{f}=0.00$ ) is instead localized on the acceptor, therefore classified as $L A$ 
(Figure 3). On the contrary, for the cluster with PCBM in on-edge configuration (4_8T/1_PCBM:e), the strongest transition is to $S_{23}(3.06 \mathrm{eV}, \mathrm{f}=5.72)$, which is similar in energy and character to $\mathrm{S}_{13}$ of 4_8T/1_PCBM:t, and to $\mathrm{S}_{4}$ of 4_8T. The manifold of CT states, in particular the one at the bottom of the band $\left(\mathrm{S}_{1}=2.51\right.$ $e V, f=0.01)$ and the first one below the active state $\left(S_{21}=3.04, f=0.18\right)$, see $\underline{\text { Figure 3 }}$, have spatially separated hole-electron densities, which are localized on the oligothiophene and PCBM domains, respectively.

Different from the on-top configuration, the first CT state is stabilized at the bottom of the band in the on-edge configuration. From the analysis of the electron-hole density distributions, we see that for the on-top configuration, the hole density is localized on the polythiophene chain which is closest to the PCBM, while for the on-edge configuration the hole density is delocalized intermolecularly across the polythiophene chains, over the whole cluster. This higher degree of delocalization causes the CT states to be stabilized and pushed below the bright state in the on-edge case (Figure 2 and Figure 3 ).

In this frame, we can rationalize the photophysical mechanisms ruling the photovoltaic processes in polymer/fullerene systems and deduce general structure-property guidelines. In $\underline{\text { Scheme } 1}$ we sketch the morphology, the excited-state energy diagram, the absorption spectrum and possible photodeactivation pathways for generic on-top and on-edge polymer/PCBM interfaces, similar to those modeled in this study (Figure 1, Figure 2 and Figure 3). 
ON TOP

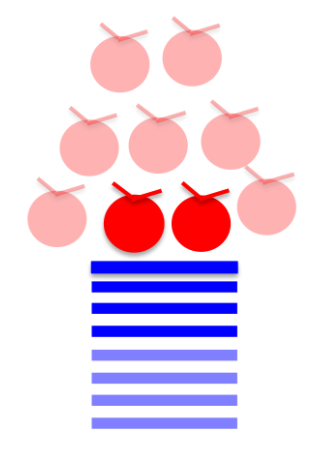

ON EDGE

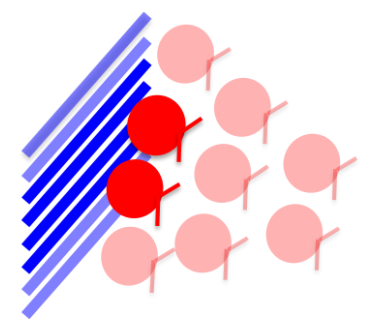

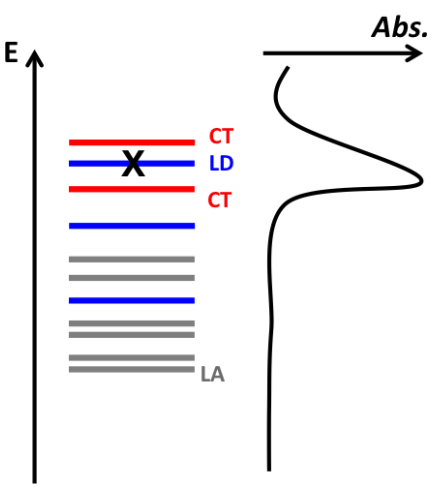

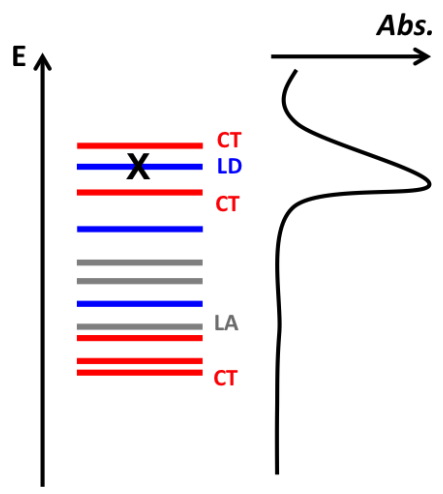

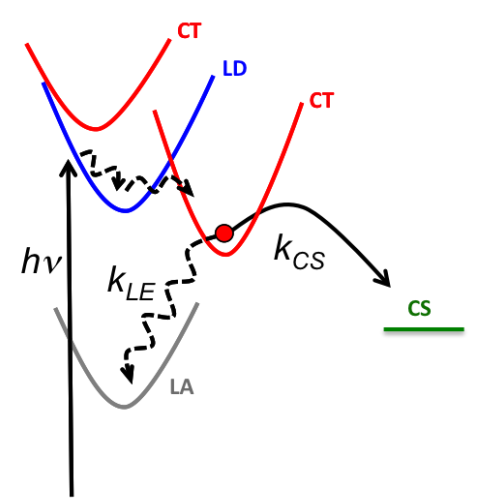

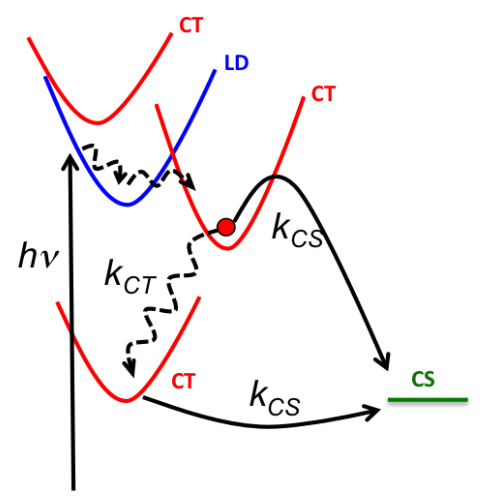

Scheme 1 Sketches of polymer/fullerene-like D/A domains in the on-top and onedge configurations, together with a representation of the excited-state energies, the state classifications, and absorption spectra as derived from our computational investigation. Schemes of possible deactivation mechanisms are also indicated, with $k_{L E}$ and $k_{C S}$ denoting the transfer rate to the localized exciton (LE) and the charge separated (CS) state, respectively.

When the PCBM is on-top of the polymer domain, CT states lie within the optical gap (in quasi-resonance with the bright $L D$ states), and thus they can be directly populated (e.g. by laser pump). Thereafter, two pathways are possible: 1) the electron and hole can further spread out, driven by nuclear dynamics ${ }^{10-11,}$ 35 or by a favorable repulsive electrostatic potential, ${ }^{18 a}$ as possibly induced by crystalline phases or $\pi$-electron delocalization, ${ }^{8}$ leading to an effective charge separation process, or 2) IC can occur, $35 \mathrm{~b}$ transferring the excitation to unproductive localized states ( $L A$ on PCBM) at the bottom of the band. The latter can be interpreted as a loss mechanism leading to geminate recombination. 
In the case of an on-edge PCBM configuration, the dipole-allowed excited state(s) and, consequently, the absorption spectrum are similar to the on-top case but the underlying excited-state electronic structure of the interface is different. The CT states are now spread throughout the band, affecting the photophysical properties. Similarly to the on-top case, the photovoltaic mechanisms can involve: 1) direct optical population of CT states ${ }^{13}$ or 2) IC processes. ${ }^{10,35 b}$ However, contrary to the on-top case, the IC processes (i.e. thermalization) can be productive in the on-edge configuration, because the states at the bottom of the band have CT character, possibly leading to charge separation. This scheme not only suggests a possible interpretation of the charge generation mechanism upon photoexcitation below the optical gap (i.e. cold charge generation), but also provides a justification for the observed delayed charge separation as coming from relaxed CT states at the interface.7,16b

We consider our findings relevant because we can now explain the longdebated OPV charge generation processes at the atomistic level.2a, 6, 26 The simultaneous presence of mixed on-top and on-edge phases in the morphology does not alter the optical absorption spectrum of the blend but does affect the nature of the excited states at the interface, and consequently the photophysics. Observed phenomena such as $i$ ) the simultaneous charge generation obtained from above (hot) ${ }^{9}$ and below (cold) optical-gap excitation, ${ }^{7,36}$ ii) the instantaneous and delayed charge separation, as caused by exciton-diffusion limited charge generation or slow separation of CT states, ${ }^{16 \mathrm{~b}}$ and $\left.i i i\right)$ the pumppush-probe charge generation, i.e. charges extracted after re-excitation (push), ${ }^{1}$ can now be rationalized based on our computations, by taking into account the excited states of both the on-top and on-edge configurations, at the same time. 
Regardless of the computational methods used and the D/A systems studied, our conclusions are general and, furthermore, they go beyond the molecular dimer paradigm, by accessing a manifold of excited states for the description of the photophysics of microscopic D/A clusters. We believe that these model clusters can be considered as realistic interfacial domains for the description of polymer/PCBM photovoltaic processes and that they can be used in the future, in combination with embedding methodologies (e.g. QM/MM), to further explore the effects of the environment on the excited states ${ }^{37}$ in extended, realistic, organic/organic interfaces.

\section{ASSOCIATED CONTENT}

\section{Supporting Information}

Vertical excitation energies computed at the TD- $\omega$ B97X-D/6-31G* level for homogeneous clusters. Excited-state classification for each cluster investigated in the manuscript. Excited-state classification for large clusters containing two PCBM molecules. $S_{1}$ optimized structure and state characterization for 1_8T/1_PCBM:t. Comparison between vertical excitations of 4_8T/1_PCBM clusters computed with several approaches (gas phase, C-PCM, range-separated functional with optimized $\omega$ value).

\section{AUTHORS INFORMATION}

\section{Corresponding Authors}

*D. F.: E-mail: fazzi@kofo.mpg.de

*M. B.: E-mail: mario.barbatti@univ-amu.fr

*W. T.: E-mail: thiel@kofo.mpg.de 


\section{ORCID}

Daniele Fazzi: 0000-0002-8515-4214

Mario Barbatti: 0000-0001-9336-6607

Walter Thiel: 0000-0001-6780-0350

\section{Notes}

The authors declare no competing financial interest.

\section{ACKNOWLEDGEMENTS}

MB work was supported by Excellence Initiative of Aix-Marseille University (A*MIDEX) and the project Equip@Meso (ANR-10-EQPX-29-01), both funded by the French Government "Investissements d'Avenir" program. MB also acknowledges funding from HPC resources from GENCI-CINES (Grant 2017A0010810012).

\section{REFERENCES}

1. Jean, J.; Brown, P. R.; Jaffe, R. L.; Buonassisi, T.; Bulović, V. Pathways for solar photovoltaics. Energy Environ. Sci. 2015, 8 (4), 1200-1219.

2. (a) Savoie, B. M.; Jackson, N. E.; Chen, L. X.; Marks, T. J.; Ratner, M. A. Mesoscopic Features of Charge Generation in Organic Semiconductors. Acc. Chem. Res. 2014, 47, 3385-3394; (b) Clarke, T. M.; Durrant, J. R., Charge Photogeneration in Organic Solar Cells. Chem. Rev. 2010, 110 (11), 6736-6767; (c) Bakulin, A. A.; Rao, A.; Pavelyev, V. G.; van Loosdrecht, P. H.; Pshenichnikov, M. S.; Niedzialek, D.; Cornil, J.; Beljonne, D.; Friend, R. H. The role of driving energy and delocalized States for charge separation in organic semiconductors. Science 2012, 335 (6074), 1340-1344.

3. Brédas, J.L.; Norton, J. E.; Cornil, J.; Coropceanu, V. Molecular Understanding of Organic Solar Cells: The Challenges. Acc. Chem. Res. 2009, 42, 1691-1699.

4. Hedley, G. J.; Ruseckas, A.; Samuel, I. D., Light Harvesting for Organic Photovoltaics. Chem. Rev. 2017, 117 (2), 796-837. 
5. (a) Tamura, H.; Burghardt, I., Potential Barrier and Excess Energy for Electron-Hole Separation from the Charge-Transfer Exciton at Donor-Acceptor Heterojunctions of Organic Solar Cells. J. Phys. Chem. C 2013, 117 (29), 1502015025; (b) Tamura, H.; Burghardt, I., Ultrafast charge separation in organic photovoltaics enhanced by charge delocalization and vibronically hot exciton dissociation. J. Am. Chem. Soc. 2013, 135 (44), 16364-16367.

6. (a) Brückner, C.; Würthner, F.; Meerholz, K.; Engels, B., StructureProperty Relationships from Atomistic Multiscale Simulations of the Relevant Processes in Organic Solar Cells. I. Thermodynamic Aspects. J. Phys. Chem. C 2017, 121 (1), 4-25; (b) Brückner, C.; Würthner, F.; Meerholz, K.; Engels, B., Atomistic Approach To Simulate Processes Relevant for the Efficiencies of Organic Solar Cells as a Function of Molecular Properties. II. Kinetic Aspects. J. Phys. Chem. C 2017, 121 (1), 26-51; (c) Casalegno, M.; Pastore, R.; Idé, J.; Po, R.; Raos, G., Origin of Charge Separation at Organic Photovoltaic Heterojunctions: A Mesoscale Quantum Mechanical View. J. Phys. Chem. C 2017.

7. Vandewal, K.; Albrecht, S.; Hoke, E. T.; Graham, K. R.; Widmer, J.; Douglas, J. D.; Schubert, M.; Mateker, W. R.; Bloking, J. T.; Burkhard, G. F.; Sellinger, A.; Frechet, J. M.; Amassian, A.; Riede, M. K.; McGehee, M. D.; Neher, D.; Salleo, A., Efficient charge generation by relaxed charge-transfer states at organic interfaces. Nat. Mater. 2014, 13 (1), 63-68.

8. Gelinas, S.; Rao, A.; Kumar, A.; Smith, S. L.; Chin, A. W.; Clark, J.; van der Poll, T. S.; Bazan, G. C.; Friend, R. H., Ultrafast long-range charge separation in organic semiconductor photovoltaic diodes. Science 2014, 343 (6170), 512-516.

9. Grancini, G.; Maiuri, M.; Fazzi, D.; Petrozza, A.; Egelhaaf, H. J.; Brida, D.; Cerullo, G.; Lanzani, G., Hot exciton dissociation in polymer solar cells. Nat. Mater. 2013, 12 (1), 29-33.

10. Fazzi, D.; Barbatti, M.; Thiel, W., Unveiling the Role of Hot Charge-Transfer States in Molecular Aggregates via Nonadiabatic Dynamics. J. Am. Chem. Soc. 2016, 138 (13), 4502-11.

11. Bittner, E. R.; Silva, C., Noise-induced quantum coherence drives photocarrier generation dynamics at polymeric semiconductor heterojunctions. Nature Comm. 2014, 5, 3119.

12. Caruso, D.; Troisi, A., Long-range exciton dissociation in organic solar cells. Proc. Natl. Acad. Sci. 2012, 109 (34), 13498-13502.

13. Ma, H.; Troisi, A., Direct optical generation of long-range charge-transfer States in organic photovoltaics. Adv. Mater. 2014, 26 (35), 6163-6167.

14. Roland, T.; Leonard, J.; Hernandez Ramirez, G.; Mery, S.; Yurchenko, O.; Ludwigs, S.; Haacke, S., Sub-100 fs charge transfer in a novel donor-acceptordonor triad organized in a smectic film. Phys. Chem. Chem. Phys. 2012, 14 (1), 273-279. 
15. Wenzel, J.; Dreuw, A.; Burghardt, I., Charge and energy transfer in a bithiophene perylenediimide based donor-acceptor-donor system for use in organic photovoltaics. Phys. Chem. Chem. Phys. 2013, 15 (28), 11704-11716.

16. (a) Chen, K.; Barker, A. J.; Reish, M. E.; Gordon, K. C.; Hodgkiss, J. M., Broadband ultrafast photoluminescence spectroscopy resolves charge photogeneration via delocalized hot excitons in polymer:fullerene photovoltaic blends. J. Am. Chem. Soc. 2013, 135 (49), 18502-18512; (b) Barker, A. J.; Chen, K.; Hodgkiss, J. M., Distance distributions of photogenerated charge pairs in organic photovoltaic cells. J. Am. Chem. Soc. 2014, 136 (34), 12018-12026.

17. Sen, K.; Crespo-Otero, R.; Weingart, O.; Thiel, W.; Barbatti, M., Interfacial States in Donor-Acceptor Organic Heterojunctions: Computational Insights into Thiophene-Oligomer/Fullerene Junctions. J. Chem. Theory Comput. 2013, 9 (1), 533-542.

18. (a) Castet, F.; D'Avino, G.; Muccioli, L.; Cornil, J.; Beljonne, D., Charge separation energetics at organic heterojunctions: on the role of structural and electrostatic disorder. Phys. Chem. Chem. Phys 2014, 16 (38), 20279-20290; (b) Poelking, C.; Andrienko, D., Long-Range Embedding of Molecular Ions and Excitations in a Polarizable Molecular Environment. J. Chem. Theory Comput. 2016, 12 (9), 4516-4523.

19. (a) D’Avino, G.; Mothy, S.; Muccioli, L.; Zannoni, C.; Wang, L.; Cornil, J.; Beljonne, D.; Castet, F., Energetics of Electron-Hole Separation at P3HT/PCBM Heterojunctions. The Journal of Physical Chemistry C 2013, 117 (25), 1298112990; (b) Grancini, G.; Polli, D.; Fazzi, D.; Cabanillas-Gonzalez, J.; Cerullo, G.; Lanzani, G. Transient Absorption Imaging of P3HT:PCBM Photovoltaic Blend: Evidence For Interfacial Charge Transfer State. J. Phys. Chem. Lett. 2011, 2 (9), 1099-1105; (c) Kanai, Y.; Grossman, J. C. Insights on Interfacial Charge Transfer Across P3HT/Fullerene Photovoltaic Heterojunction from Ab Initio Calculations. Nano Letters 2007, 7, 1967-1972.

20. (a) Zheng, Z.; Bredas, J. L.; Coropceanu, V., Description of the Charge Transfer States at the Pentacene/C60 Interface: Combining Range-Separated Hybrid Functionals with the Polarizable Continuum Model. J. Phys. Chem. Lett. 2016, 7 (13), 2616-2621; (b) Zheng, Z.; Tummala, N. R.; Fu, Y. T.; Coropceanu, V.; Bredas, J. L., Charge-Transfer States in Organic Solar Cells: Understanding the Impact of Polarization, Delocalization, and Disorder. ACS Appl. Mater. Interfaces 2017, 9 (21), 18095-18102; (c) Zhang, C.-R.; Sears, J. S.; Yang, B.; Aziz, S. G.; Coropceanu, V.; Brédas, J.-L., Theoretical Study of the Local and Charge-Transfer Excitations in Model Complexes of Pentacene-C60Using Tuned Range-Separated Hybrid Functionals. J. Chem. Theory Comput. 2014, 10 (6), 2379-2388.

21. Baumeier, B.; Rohlfing, M.; Andrienko, D., Electronic Excitations in PushPull Oligomers and Their Complexes with Fullerene from Many-Body Green's Functions Theory with Polarizable Embedding. J. Chem. Theory Comput. 2014, 10 (8), 3104-3110. 
22. (a) Baumeier, B.; Andrienko, D.; Rohlfing, M., Frenkel and Charge-Transfer Excitations in Donor-acceptor Complexes from Many-Body Green's Functions Theory. J. Chem. Theory and Comput. 2012, 8 (8), 2790-2795; (b) Rozzi, C. A.; Falke, S. M.; Spallanzani, N.; Rubio, A.; Molinari, E.; Brida, D.; Maiuri, M.; Cerullo, G.; Schramm, H.; Christoffers, J.; Lienau, C., Quantum coherence controls the charge separation in a prototypical artificial light-harvesting system. Nature Comm. 2013, 4, 1602-1608.

23. Zheng, Z.; Egger, D. A.; Bredas, J. L.; Kronik, L.; Coropceanu, V., Effect of Solid-State Polarization on Charge-Transfer Excitations and Transport Levels at Organic Interfaces from a Screened Range-Separated Hybrid Functional. J. Phys. Chem. Lett. 2017, 3277-3283.

24. Duchemin, I.; Blase, X., Resonant hot charge-transfer excitations in fullerene-porphyrin complexes: Many-body Bethe-Salpeter study. Phys. Rev. $B$ 2013, 87, 245412.

25. Borges, I., Jr.; Aquino, A. J.; Kohn, A.; Nieman, R.; Hase, W. L.; Chen, L. X.; Lischka, $\mathrm{H}$., Ab initio modeling of excitonic and charge-transfer states in organic semiconductors: the PTB1/PCBM low band gap system. J. Am. Chem. Soc. 2013, 135 (49), 18252-18255.

26. Niedzialek, D.; Duchemin, I.; de Queiroz, T. B.; Osella, S.; Rao, A.; Friend, R.; Blase, X.; Kümmel, S.; Beljonne, D., First Principles Calculations of Charge Transfer Excitations in Polymer-Fullerene Complexes: Influence of Excess Energy. Adv. Funct. Mater. 2015, 25 (13), 1972-1984.

27. Gaussian 09, Revision D.01, M. J. Frisch, G. W. Trucks, H. B. Schlegel, G. E. Scuseria, M. A. Robb, J. R. Cheeseman, G. Scalmani, V. Barone, G. A. Petersson, H. Nakatsuji, X. Li, M. Caricato, A. Marenich, J. Bloino, B. G. Janesko, R. Gomperts, B. Mennucci, H. P. Hratchian, J. V. Ortiz, A. F. Izmaylov, J. L. Sonnenberg, D. WilliamsYoung, F. Ding, F. Lipparini, F. Egidi, J. Goings, B. Peng, A. Petrone, T. Henderson, D. Ranasinghe, V. G. Zakrzewski, J. Gao, N. Rega, G. Zheng, W. Liang, M. Hada, M. Ehara, K. Toyota, R. Fukuda, J. Hasegawa, M. Ishida, T. Nakajima, Y. Honda, O. Kitao, H. Nakai, T. Vreven, K. Throssell, J. A. Montgomery, Jr., J. E. Peralta, F. Ogliaro, M. Bearpark, J. J. Heyd, E. Brothers, K. N. Kudin, V. N. Staroverov, T. Keith, R. Kobayashi, J. Normand, K. Raghavachari, A. Rendell, J. C. Burant, S. S. Iyengar, J. Tomasi, M. Cossi, J. M. Millam, M. Klene, C. Adamo, R. Cammi, J. W. Ochterski, R. L. Martin, K. Morokuma, O. Farkas, J. B. Foresman, and D. J. Fox, Gaussian, Inc., Wallingford CT, 2016.

28. Refaely-Abramson, S.; Sharifzadeh, S.; Jain, M.; Baer, R.; Neaton, J. B.; Kronik, L., Gap renormalization of molecular crystals from density-functional theory. Phys. Rev. B 2013, 88 (8), 081204.

29. de Queiroz, T. B.; Kummel, S., Tuned range separated hybrid functionals for solvated low bandgap oligomers. J. Chem. Phys. 2015, 143 (3), 034101. 
30. Kronik, L.; Stein, T.; Refaely-Abramson, S.; Baer, R., Excitation Gaps of Finite-Sized Systems from Optimally Tuned Range-Separated Hybrid Functionals. J. Chem. Theory Comput. 2012, 8 (5), 1515-1531.

31. (a) Plasser, F.; Wormit, M.; Dreuw, A., New tools for the systematic analysis and visualization of electronic excitations. I. Formalism. J. Chem. Phys. 2014, 141 (2), 024106; (b) Mewes, S. A.; Plasser, F.; Dreuw, A., Communication: Exciton analysis in time-dependent density functional theory: How functionals shape excited-state characters. J Chem. Phys. 2015, 143 (17), 171101; (c) Plasser, F.; Thomitzni, B.; Bappler, S. A.; Wenzel, J.; Rehn, D. R.; Wormit, M.; Dreuw, A., Statistical analysis of electronic excitation processes: Spatial location, compactness, charge transfer, and electron-hole correlation. J. Comput. Chem. 2015, 36 (21), 1609-1620.

32. Crespo-Otero, R.; Barbatti, M., Spectrum simulation and decomposition with nuclear ensemble: formal derivation and application to benzene, furan and 2-phenylfuran. Theo. Chem. Acc. 2012, 131 (6), 1237.

33. Fazzi, D.; Grancini, G.; Maiuri, M.; Brida, D.; Cerullo, G.; Lanzani, G., Ultrafast internal conversion in a low band gap polymer for photovoltaics: experimental and theoretical study. Physical chemistry chemical physics : PCCP 2012, 14 (18), 6367-6374.

34. We have optimized at the TD-DFT level the first excited state (S1) of a minimal D/A interface, i.e. 1_8T/1_PCBM. The electron-hole denisty distribution results to be entirely localized on the acceptor PCBM. This aspect remarks that, even in the relaxed state at the bottom of the band, the exciton is localized on the acceptor.

35. (a) Fazzi, D.; Barbatti, M.; Thiel, W., Modeling ultrafast exciton deactivation in oligothiophenes via nonadiabatic dynamics. Physical chemistry chemical physics : PCCP 2015, 17 (12), 7787-99; (b) Jailaubekov, A. E.; Willard, A. P.; Tritsch, J. R.; Chan, W. L.; Sai, N.; Gearba, R.; Kaake, L. G.; Williams, K. J.; Leung, K.; Rossky, P. J.; Zhu, X. Y., Hot charge-transfer excitons set the time limit for charge separation at donor/acceptor interfaces in organic photovoltaics. Nature materials 2013, 12 (1), 66-73.

36. Jiye Lee, K. V., Shane R. Yost, Matthias E. Bahlke, Ludwig Goris, Marc A. Baldo, Jean V. Manca, and Troy Van Voorhis, Charge Transfer State Versus Hot Exciton Dissociation in Polymer-Fullerene Blended Solar Cells. J. Am. Chem. Soc. 2010, 132, 3 .

37. Mennucci, B.; Modeling environment effects on spectroscopies through QM/classical models, Phys. Chem. Chem. Phys. 2013, 15, 6583-6594. 